\title{
Parameterized families of quadratic number fields with 3-rank at least 2
}

\author{
by \\ Carl Erickson (Stanford, CA), Nathan Kaplan (Princeton, NJ), \\ Neil Mendoza (Williamstown, MA), \\ Allison M. PACELli (Williamstown, MA) \\ and Todd Shayler (Williamstown, MA)
}

1. Introduction. It is well known that there are infinitely many quadratic number fields with class number divisible by a given integer $n$ (see Nagell [8] (1922) for imaginary fields and Yamamoto [11] (1970) and Weinberger [10] (1973) for real fields). A related question concerns the $n$-rank of the field, that is, the greatest integer $r$ for which the class group contains a subgroup isomorphic to $(\mathbb{Z} / n \mathbb{Z})^{r}$. In [11], Yamamoto showed that infinitely many imaginary quadratic number fields have $n$-rank $\geq 2$ for any positive integer $n \geq 2$. In 1978, Diaz y Diaz [2] developed an algorithm for generating imaginary quadratic fields with 3-rank at least 2, and Craig [1] showed in 1973 that there are infinitely many real quadratic number fields with 3-rank at least 2 and infinitely many imaginary quadratic number fields with 3 -rank at least 3. A few examples of higher 3-rank have also been found (see for instance Llorente and Quer [6,9] who found in 1987/1988 three imaginary quadratic number fields with 3-rank 6). In this paper, we give infinite, simply parameterized families of real and imaginary quadratic fields with 3-rank 2. Although the existence of such fields has been known, the fields here are much easier to describe, and the parameterization yields a new lower bound on the number of fields with discriminant $<x$ and 3 -rank $\geq 2$ (see [7]).

The main result is as follows:

TheOREM 1.1. Let $w \equiv \pm 1(\bmod 6)$, and let $c$ be any integer with $c \equiv w$ $(\bmod 6)$. Then

$$
\mathbb{Q}\left(\sqrt{c\left(w^{2}+18 c w+108 c^{2}\right)\left(4 w^{3}-27 c w^{2}-486 c^{2} w-2916 c^{3}\right)}\right)
$$

has 3 -rank at least 2 .

2000 Mathematics Subject Classification: Primary 11R11.

Key words and phrases: 3-rank, class number, class group, quadratic number field. 
Notice that if $c$ and $w$ are relatively prime, and $p$ is an odd prime with $p^{2 a+1} \| c$ for some non-negative integer $a$, then $p$ is ramified. The parameterization therefore yields infinitely many real and infinitely many imaginary quadratic fields since only finitely many primes are ramified in a given field.

As a special case of the theorem where $c=1$ and $w=6 a+1$, we have the following:

Corollary 1.2. For any integer a, $\mathbb{Q}(\sqrt{f(a)})$ has 3-rank at least 2, where

$$
\begin{aligned}
f(a) & =31104 a^{5}+84240 a^{4}-69120 a^{3}-572040 a^{2}-813336 a-434975 \\
& =\left(36 a^{2}+120 a+127\right)\left(864 a^{3}-540 a^{2}-3168 a-3425\right) .
\end{aligned}
$$

It is not hard to show that this special case itself yields infinitely many real quadratic fields and infinitely many imaginary fields.

The idea of the proof is to construct, for each $d$ of the prescribed form, two distinct unramified, cyclic, cubic extensions of $\mathbb{Q}(\sqrt{d})$. By class field theory, then, the field has 3-rank at least 2. We use Kishi and Miyake's [4] characterization of quadratic number fields with class number divisible by 3 to construct two such extensions of the same quadratic field $\mathbb{Q}(\sqrt{d})$; we guarantee that the fields are distinct by showing that the prime 3 decomposes differently in each.

2. Proof. Recall that the Hilbert class field of a number field $K$ is the maximal unramified abelian extension of $K$, and that $\operatorname{Gal}(H / K) \cong \mathrm{Cl}_{K}$, where $\mathrm{Cl}_{K}$ denotes the ideal class group of $K$. It follows that the class number of $K$ is divisible by 3 if and only if there is a cyclic, cubic, unramified extension of $K$. In fact, Hasse's theorem [3] states that if $K$ is a quadratic field, then $K$ has 3-rank $n$ if and only if there are exactly $\left(3^{n}-1\right) / 2$ cyclic, cubic, unramified extensions of $K$. To prove that a quadratic field $K$ has 3 -rank at least 2, therefore, it suffices to show that $K$ has two distinct cyclic, cubic, unramified extensions.

First, notice that we may assume that $c$ and $w$ are relatively prime, because the quadratic field parameterized by $c$ and $w$ is the same as the field parameterized by $c /(c, w)$ and $w /(c, w)$.

In [4], Kishi and Miyake give the following characterization of all quadratic fields with class number divisible by 3 .

Theorem 2.1. Choose $u, w \in \mathbb{Z}$ and $\operatorname{let} g(Z)=Z^{3}-u w Z-u^{2}$. If

(i) $d=4 u w^{3}-27 u^{2}$ is not a square in $\mathbb{Z}$,

(ii) $u$ and $w$ are relatively prime,

(iii) $g(Z)$ is irreducible,

(iv) one of the following conditions holds: 
(I) $3 \nmid w$,

(II) $3 \mid w, u w \not \equiv 3(\bmod 9), u \equiv w \pm 1(\bmod 9)$

(III) $3 \mid w, u w \equiv 3(\bmod 9), u \equiv w \pm 1(\bmod 27)$,

then the normal closure of $\mathbb{Q}(\theta)$, where $\theta$ is a root of $g(Z)$, is a cyclic, cubic, unramified extension of $\mathbb{Q}(\sqrt{d})$; in particular, then, $K=\mathbb{Q}(\sqrt{d})$ has class number divisible by 3 . Conversely, every quadratic number field $K$ with class number divisible by 3 and every unramified, cyclic, cubic extension of $K$ is given by a suitable choice of integers $u$ and $w$.

Given integers $c$ and $w$ with $c \equiv w \equiv \pm 1(\bmod 6)$, we define integers $u, x$, and $y$ so that the two pairs of integers $u, w$ and $x, y$ each satisfy the conditions of Theorem 2.1. In addition, if $\theta_{1}$ is a root of $g_{1}(Z)=Z^{3}-u w Z$ $-u^{2}$ and $\theta_{2}$ is a root of $g_{2}(Z)=Z^{3}-x y Z-x^{2}$, then the cubic fields $\mathbb{Q}\left(\theta_{1}\right)$ and $\mathbb{Q}\left(\theta_{2}\right)$ have discriminants with the same square free part as

$$
d=c\left(w^{2}+18 c w+108 c^{2}\right)\left(4 w^{3}-27 c w^{2}-486 c^{2} w-2916 c^{3}\right) .
$$

By Theorem 2.1 , then, $\mathbb{Q}(\sqrt{d})$ has two cyclic, cubic, unramified extensions $L_{1}$ and $L_{2}$ (we also show that $L_{1}$ and $L_{2}$ are distinct by showing that the prime 3 splits differently in each). It then follows from Hasse's theorem that $\mathbb{Q}(\sqrt{d})$ has 3 -rank at least 2 . Here $L_{1}$ and $L_{2}$ are the normal closures of $\mathbb{Q}\left(\theta_{1}\right)$ and $\mathbb{Q}\left(\theta_{2}\right)$; since $d$ is not a square, each has Galois group $S_{3}$ over $\mathbb{Q}$.

LEMMA 2.2. Let $c$ and $w$ be integers with $c \equiv w \equiv \pm 1(\bmod 6)$. If

$$
u=c\left(w^{2}+18 c w+108 c^{2}\right), \quad x=9 u, \quad y=w+18 c,
$$

then the pairs $u, w$ and $x, y$ each satisfy the hypotheses of Theorem 2.1, that is, $\mathbb{Q}\left(\sqrt{4 u w^{3}-27 u^{2}}\right)$ and $\mathbb{Q}\left(\sqrt{4 x y^{3}-27 x^{2}}\right)$ each admit cyclic, cubic, unramified extensions.

Proof. First note that since $c \equiv w \equiv \pm 1(\bmod 6)$ and $(c, w)=1$, we have $(6 c, w)=1$. It follows that $(u, w)=1$ since $u \equiv 108 c^{3}(\bmod w)$. Also, since

$$
x=9 c\left(w^{2}+18 c w+108 c^{2}\right)=9 c w(w+18 c)+972 c^{3} \equiv 972 c^{3}(\bmod y),
$$

and $y \equiv w(\bmod 6 c)$, we see that any prime factor of $x$ and $y$ would divide $6 c$ and therefore $w$. Since $(6 c, w)=1$, this implies that $(x, y)=1$ as well. Thus condition (ii) in Theorem 2.1 is satisfied.

For condition (iii), observe that $c$ and $w$ are odd, so $u, x$, and $y$ are odd as well. Then

$$
\begin{aligned}
g_{1}(Z) & =Z^{3}-u w Z-u^{2} \equiv Z^{3}+Z+1 \\
& \equiv Z^{3}-x y Z-x^{2}=g_{2}(Z)(\bmod 2),
\end{aligned}
$$

so $g_{1}$ and $g_{2}$ are both irreducible over $\mathbb{Z}$.

Condition (iv) is clearly satisfied since $w$ and therefore $y$ are not divisible by 3 . 
Finally, we show that condition (i) is also satisfied, namely, that $4 u w^{3}-$ $27 u^{2}$ and $4 x y^{3}-27 x^{2}$ are not squares in $\mathbb{Z}$. This follows, in fact, from the other conditions. Let $\theta_{1}$ and $\theta_{2}$ be roots of $g_{1}(Z)$ and $g_{2}(Z)$, respectively, and let $L_{1}$ and $L_{2}$ be the normal closures of $\mathbb{Q}\left(\theta_{1}\right)$ and $\mathbb{Q}\left(\theta_{2}\right)$, respectively. It suffices to show that the Galois groups of $L_{1}$ and $L_{2}$ over $\mathbb{Q}$ are $S_{3}$ since cubic fields with square discriminants are normal. So let $i=1,2$ and suppose, for contradiction, that the Galois group of $L_{i}$ over $\mathbb{Q}$ is $\mathbb{Z} / 3 \mathbb{Z}$. Let $p$ be a prime in $\mathbb{Z}$ that is totally ramified in $L_{i}$. If $v_{p}(a)$ denotes the exact power of $p$ dividing $a$, then Llorente and Nart's characterization of prime decomposition in cubic fields [5] implies that either

(1) $1 \leq v_{p}\left(b_{i}\right) \leq v_{p}\left(a_{i}\right)$, where $g_{i}^{*}(Z)=Z^{3}+a_{i} Z+b_{i}$ is obtained from $g_{i}(Z)$ by substituting $Z / t$ for $Z$ with appropriate $t \in \mathbb{Z}$ so that $v_{q}\left(a_{i}\right) \leq 1$ or $v_{q}\left(b_{i}\right) \leq 2$ for all primes $q$,

or

(2) $p=3,3 \mid a_{i}$.

If $p \nmid u w$, then clearly the first condition does not hold. If $p \mid w$, then $v_{p}\left(b_{i}\right)=0$ since $u$ and $w$ are relatively prime, so the first condition cannot hold for $i=1$ or 2 . Neither can it hold if $p \mid u$, for then as in [4, Lemma 2] we see that $v_{p}\left(a_{i}\right)=\beta$ and $v_{p}\left(b_{i}\right)=n+2 \beta$ for some integers $n$ and $\beta$, with $\beta=0$ or 1 , where $v_{p}(u)=2 n+\beta$ (resp. $\left.v_{p}(x)=2 n+\beta\right)$. The second condition is impossible for $i=1$, because $3 \nmid c w$ and therefore $3 \nmid a_{1}$. If $i=2$, after substitution (with $t=3), v_{3}\left(a_{1}\right)=v_{3}(u(w+18 c))=0$, so the second condition does not hold. Thus, no prime is totally ramified in $L_{1}$, contradicting the assumption that the splitting field of $g_{1}(Z)$ is a $\mathbb{Z}_{3}$-extension of $\mathbb{Q}$. The argument for $L_{2}$ is similar. The pairs $u, w$ and $x, y$ must therefore each generate cubic, cyclic, unramified extensions of the quadratic fields $\mathbb{Q}\left(\sqrt{4 u w^{3}-27 u^{2}}\right)$ and $\mathbb{Q}\left(\sqrt{4 x y^{3}-27 x^{2}}\right)$, respectively.

The following lemma follows from Theorem 1 in Llorente and Nart [5].

Lemma 2.3. For $u, w \in \mathbb{Z}$, set $g(Z)=Z^{3}-u w Z-u^{2}$, and let $\theta$ be $a$ root of $g$.

(i) If $u w \equiv 1(\bmod 3)$, then 3 is inert in $\mathbb{Q}(\theta)$.

(ii) If $v_{3}(x)=2 n$ for some $n>0$ with $x y / 3^{2 n} \equiv 1(\bmod 3)$, then 3 splits completely in $\mathbb{Q}(\theta)$.

We are now ready to prove the main theorem.

Proof of Theorem 1.1. Given $c \equiv w \equiv \pm 1(\bmod 6)$, set

$$
u=c\left(w^{2}+18 c w+108 c^{2}\right), \quad x=9 u, \quad y=w+18 c .
$$

Let $\theta_{1}$ be a root of $g_{1}(Z)=Z^{3}-u w Z-u^{2}$ and $\theta_{2}$ a root of $g_{2}(Z)=$ $Z^{3}-x y Z-x^{2}$. Let $L_{1}$ and $L_{2}$ denote the normal closures of $\mathbb{Q}\left(\theta_{1}\right)$ and $\mathbb{Q}\left(\theta_{2}\right)$, 
respectively. By Lemma 2.2, the pairs $u, w$ and $x, y$ satisfy the hypotheses of Theorem 2.1, so that $L_{1}$ and $L_{2}$ are unramified, cyclic, cubic extensions of $\mathbb{Q}\left(\theta_{1}\right)$ and $\mathbb{Q}\left(\theta_{2}\right)$, respectively. Notice, however, that the cubic fields $\mathbb{Q}\left(\theta_{1}\right)$ and $\mathbb{Q}\left(\theta_{2}\right)$ have discriminants which differ by a square factor:

$$
\begin{aligned}
4 x y^{3}-27 x^{2} & =4(9 u)(w+18 c)^{3}-27(9 u)^{2} \\
& =9\left[4 u\left(w^{3}+54 c\left(w^{2}+18 w c+108 c^{2}\right)\right)-243 u^{2}\right] \\
& =9\left[4 u\left(w^{3}+54 u\right)-243 u^{2}\right]=9\left(4 u w^{3}-27 u^{2}\right) .
\end{aligned}
$$

Thus $L_{1}$ and $L_{2}$ are both $S_{3}$-extensions of $\mathbb{Q}$ with the same quadratic subfield $\mathbb{Q}(\sqrt{d})$, where

$$
\begin{aligned}
d & =\sqrt{4 u w^{3}-27 u^{2}} \\
& =\sqrt{c\left(w^{2}+18 c w+108 c^{2}\right)\left(w^{3}-27 c w^{2}-486 c^{2} w-2916 c^{3}\right)} .
\end{aligned}
$$

Finally, we claim that $L_{1}$ and $L_{2}$ are not isomorphic. We will show that the prime 3 splits differently in the two fields. Since $v_{3}(x)=2$, and $x y / 9=$ $u(w+18 c) \equiv u w \equiv 1(\bmod 3)$, Lemma 2.3 shows that 3 splits completely in $\mathbb{Q}\left(\theta_{2}\right)$. It follows that 3 must also split completely in its normal closure $L_{2}$. Since $u w \equiv 1(\bmod 3)$, Lemma 2.3 implies that 3 is inert in $\mathbb{Q}\left(\theta_{1}\right)$. Thus 3 does not split completely in $L_{1}$ (in fact, 3 must factor as the product of two distinct primes in $L_{1}$ ), and so $L_{1}$ and $L_{2}$ are not isomorphic. Thus $\mathbb{Q}(\sqrt{d})$ has two distinct cubic, cyclic, unramified extensions, and therefore has 3 -rank at least 2 .

Proof of Corollary 1.2. The given example results from letting $c=1$ and writing $w=6 a+1$ for some integer $a$. Then $u=w^{2}+18 w+108=$ $36 a^{2}+120 a+127$ and $4 w^{3}-27 u=864 a^{3}-540 a^{2}-3168 a-3425$. We will show that the family above gives infinitely many imaginary quadratic number fields with 3-rank at least 2 and infinitely many real quadratic number fields with 3 -rank at least 2 . To see that this is the case, let $p$ be any prime with $p \equiv 1(\bmod 3)$. We claim that there exists some integer $a$ such that $f(a)$ is positive and $p$ divides $f(a)$ an odd number of times. Thus $p$ divides the discriminant of $\mathbb{Q}(\sqrt{f(a)})$. Since there are infinitely many primes $p \equiv 1$ $(\bmod 3)$, and only finitely many primes can divide a given discriminant, it follows that there are infinitely many real quadratic fields of the form $\mathbb{Q}(\sqrt{f(a)})$ with 3 -rank at least 2 . The same is true for negative $f(a)$, giving the same result for imaginary quadratic fields of the form $\mathbb{Q}(\sqrt{f(a)})$ with 3 -rank at least 2.

Let $p$ be any prime with $p \equiv 1(\bmod 3)$. Then -3 is a square $\bmod p$, so there exists some $z \in \mathbb{Z}$ such that $z^{2} \equiv-27(\bmod p)$. Choose $a^{\prime} \in \mathbb{Z}$ with

$$
6 a^{\prime} \equiv z-10(\bmod p) \text {. }
$$


Choose an integer $b$ with $\left(72 a^{\prime}+120\right) b \equiv 1(\bmod p)$. This is possible since

$$
72 a^{\prime}+120 \equiv 12 z(\bmod p)
$$

which implies that $\left(p, 72 a^{\prime}+120\right)=1$. Define $a$ as follows:

$$
a= \begin{cases}a^{\prime} & \text { if }\left(6 a^{\prime}+10\right)^{2} \not \equiv-27\left(\bmod p^{2}\right), \\ a^{\prime}+b p & \text { if }\left(6 a^{\prime}+10\right)^{2} \equiv-27\left(\bmod p^{2}\right) .\end{cases}
$$

In either case, then, $(6 a+10)^{2} \not \equiv-27\left(\bmod p^{2}\right)$. It follows that $v_{p}(u)=1$, since $u=36 a^{2}+120 a+127 \equiv(6 a+10)^{2}+27 \not \equiv 0\left(\bmod p^{2}\right)$, but

$$
\begin{aligned}
u & =36 a^{2}+120 a+127 \equiv(6 a+10)^{2}+27 \equiv 6 a(6 a+20)+127 \\
& \equiv(z-10)(z+10)+127 \equiv z^{2}+27 \equiv 0(\bmod p) .
\end{aligned}
$$

Since $u$ is odd for any $a$, and $(u, w)=1$, we see that $u$ and $4 w^{3}-27 u$ are relatively prime for any $a$. So $p$ exactly divides $f(a)=u\left(4 w^{3}-27 u\right)$. This implies that $p$ divides the discriminant of $\mathbb{Q}(\sqrt{f(a)})$ exactly once, and so, $p$ is ramified in $\mathbb{Q}(\sqrt{f(a)})$, as claimed.

Note that we can always choose $a^{\prime}$ and $b$ above so that $a \leq 2$; this yields infinitely many imaginary quadratic fields with 3 -rank at least 2 . Similarly, we can choose $a^{\prime}$ and $b$ so that $a \geq 3$, so there are also infinitely many real quadratic fields with 3 -rank at least 2 .

\section{References}

[1] M. Craig, A type of class group for imaginary quadratic fields, Acta Arith. 22 (1973), 449-459.

[2] F. Diaz y Diaz, On some families of imaginary quadratic fields, Math. Comp. 32 (1978), 637-650.

[3] H. Hasse, Arithmetische Theorie der kubischen Zahlkörper auf klassenkörpertheoretischer Grundlage, Math. Z. 31 (1930), 565-582.

[4] Y. Kishi and K. Miyake, Parametrization of the quadratic fields whose class numbers are divisible by three, J. Number Theory 80 (2000), 209-217.

[5] P. Llorente and E. Nart, Effective determination of the decomposition of rational primes in a cubic field, Proc. Amer. Math. Soc. 87 (1983), 579-585.

[6] P. Llorente and J. Quer, On the 3-Sylow subgroup of the class group of quadratic fields, Math. Comp. 50 (1988), 321-333.

[7] F. Luca and A. M. Pacelli, Class groups of quadratic fields of 3-rank at least 2: Effective bounds, J. Number Theory, to appear.

[8] T. Nagel, Über die Klassenzahl imaginär-quadratischer Zahlkörper, Abh. Math. Sem. Univ. Hamburg 1 (1922), 140-150.

[9] J. Quer, Corps quadratiques de 3-rang 6 et courbes elliptiques de rang 12, C. R. Acad. Sci. Paris Sér. I Math. 305 (1987), 215-218.

[10] P. J. Weinberger, Real quadratic fields with class numbers divisible by $n$, J. Number Theory 5 (1973), 237-241. 
[11] Y. Yamamoto, On unramified Galois extensions of quadratic number fields, Osaka J. Math. 7 (1970), 57-76.

Department of Mathematics

Department of Mathematics

Stanford University

Stanford, CA 94305, U.S.A.

E-mail: cerickson@stanford.edu

Department of Mathematics

Princeton University

Williams College

Williamstown, MA 01267, U.S.A.

E-mail: Neil.Mendoza@williams.edu

Allison.Pacelli@williams.edu

Princeton, NJ 08544, U.S.A.

E-mail: nathank@princeton.edu

Todd.B.Shayler@williams.edu

Received on 5.7.2006

and in revised form on 16.11.2006 